\title{
Ueber Untersuchung und Beurtheilung von Safran, Pfeffer und Honig.
}

Von

A. Hilger in München.

\section{Zur Beurtheilung des Safrans.}

Unter den Verfälschungsmitteln des Safrans, dessen Bedeutung im klassischen Alterthum und Mittelaiter als Gewürz und Farbe schon so hervorragend war, dass gesetzliche Bestimmungen und Straten für die Safranfälscher in empfindlichster Form festgestellt wurden, werden in Lehr- und Handbüchern unter Anderem genannt: Feminell und die Griffel der Safranblüthe. Die deutschen .Vereinbarungen zur einheitlichen Untersuchung und Beurtheilung von Nahrungs- und Genussmittel“" bezeichnen im Heft II auf Seite 66, Zeile 5 von unten als Verfälschungsmittel des Safrans:

"Feminell, die Griffel der Safranblüthe; sie besitzen die

gleichen Gewebe wie die Narbe, enthalten jedoch nicht den rothen Farbstoff."

In vielen Kreisen von Sachverständigen ist die Anschauung verbreitet, dass Feminell gleichbedeutend mit den Griffeln der Safranblïthe wäre. Diesen Irrthum heute in diesem Kreise zu erwähnen, halte ich für meine Pflicht, da auf Grund meiner Erfahrungen diese falsche Erklärung von Feminell Veranlassung zu Gerichtsentscheidungen gegeben hat, bei welchen zum Schaden des Händlers entschieden wurde.

Feminell ist eine Bezeichnung für die Blïthen ron Calendula officinalis (Ringelblumen), wahrscheinlich auch anderer Compositen, welche schon allgemein in den Handelskreisen des Mittelalters bekannt war und auch in den Verordnungen der Reichsstadt Nürnberg eine Rolle spielte, indem diese Stadt eine Zeitlang einen Zusatz von Feminell (Blüthen von Calendula) zum Safran duldete.

Bei einer Neuherausgabe der deutschen Vereinbarungen dürfte daher bei dem Abschnitte "Safran" bezüglich der Bezeichnung Feminell eine klarere Fassung des Textes empfehlenswerth sein.

\section{Beobachtungen bei der Furfurolbestimmung im Pfeffer.}

Vor einigen Jahren ${ }^{1}$ ) habe ich in Gemeinschaft mit F. E. Bauer Bericht erstattet über Versuche, welche beabsichtigten, durch Bestimmung der Furfurol liefernden Körper (Pentosane) in der Pfefferfrucht den Nachweis von allzu grossen Mengen von Pfefferschalen in dem gemahlenen Pfeffer zu erbringen. Es wurde damals die Phenylhydrazin-Methode in entsprechender Ausführung verwendet, welche besonders bezüglich des vollkommenen Abschlusses der Luft,

1) Forschungsherichte über Lehensmittel n. s. w. 1896, 3, 113. 
sowie auch des Trocknens des gebildeten Osazons nicht vollkommen befriedigen konnte.

Inzwischen ist die sog. Phlorogiucin-Methode für die Pentosanbestimmung in den Vordergrund getreten und hat allgemein Verwendung in den agrikulturchemischen Laboratorien gefunden; diese hat indess für den hier vorliegenden Fall mich bis jetzt nicht befriedigen können.

Eine Arbeit, welche an mich herangetreten war, um den Gehalt an Pentosanen in Pfeffersorten des Handels kennen zu lernen, gab zu einer nochmaligen Bearbeitung der früher benutzten Furfurolbestimmung mittelst Phenylhydrazin Veranlassung, deren Ergebnisse hinsichtlich einer wesentlichen Verbesserung der Ausführung der Methode mitgetheilt werden sollen.

Folgendes Verfahren, bei dessen Ausbildung ich sehr erfolgreich durch Herrn Dr. Pfyl unterstïtzt wurde, hat sich sehr bewährt und liefert gut übereinstimmende Ergebnisse:

$5 \mathrm{~g}$ gepulverter Pfeffer werden zuvor mit Alkohol und Aether vollkommen extrahirt und darauf mit etwa $100-150 \mathrm{ccm}$ Salzsäure (von 1,06 spec. Gew.) bei $150-160^{\circ}$ der Destillation unter allmählichem Zusatz ron neuer Salzsäure, bis das Destillat $400 \mathrm{ccm}$ beträgt, unterworfen. Der Destillations-Apparat, durch welchen während der ganzen Arbeit Kohlensäure geleitet wird, ist vollkommen geschlossen.

Das Destillat wird nun mit Natronlauge vorsichtig unter Abkühlung und ununterbrochenem Rühren neutralisirt, hierauf mit Essigsäure schwach angesäuert und nun die Lösung von $2 \mathrm{~g}$ salzsaurem Phenylhydrazin, $3 \mathrm{~g}$ Natriumacetat in $20 \mathrm{ccm}$ Wasser unter beständigem Rühren und Einleiten von Kohlensäure zugesetzt. Sobald das entstandene Osazon sich zusammenballt, wird dasselbe durch ein Allihn'sches Rohr filtrirt und mit $100 \mathrm{ccm}$ Wasser nachgewaschen. Das im Allihn'schen Rohre befindliche Osazon wird in absolutem Alkohol vollkommen gelöst und diese Lösung in einer gewogenen Platinschale bei $60-70^{\circ} \mathrm{im}$ Vakuum verdunstet. Im Zusammenhange mit den Ergebnissen der Untersuchung über den Pentosangehalt des Pfeffers werde ich den Apparat, der gute Dienste geleistet hat, später noch näher beschreiben.!

\section{Zur Untersuchung und Beurtheilung des Honigs.}

Die schon bedeutend vorgerïckte Zeit zwingt mich, heute nur über den Plan und die Ausführung einer grösseren Arbeit kurz zu berichten, welche Herr Dr. Porrini aus Florenz mit Unterstïtzung von Herrn Dr. Kappeller unter meiner Leitung ausgeführt hat und welche den Zweck hatte, Honige aus Deutschland, sowie auch ausländische Honige einer eingehenden chemischen Untersuchung zu unterziehen, um weitere Erfahrungen über die Zusammensetzung der Honige und deren Beurtheilung zu gewinnen.

Bei der Beschaffung des Materiales war in liebenswürdigster Weise Herr Ad. Ke im aus Grünwald thätig, dem ich für seine Bemühungen danke. 
Zur Untersuchung kamen 24 deutsche Honige, darunter 9 Waldhonige, 22 ausländische Honige, von denen 2 aus Griechenland, 13 aus Amerika, 1 aus Spanien, 1 aus Rumänien, 3 aus Ungarn, 1 aus Norwegen nnd 1 aus Slavonien stammten, und endlich 8 Kunsthonige.

Bei jeder Honigprobe wurden bestimmt: Specifisches Gewicht der Lösung 1:2, Wasser, Zucker vor und nach der Inversion, Rohrzucker, Nichtzucker, Asche, Polarisation und freie Säure als Ameisensäure berechnet. Auch wurde bei allen Sorten festgestellt, ob dieselben mehr oder weniger Schwefelsäure enthalten; bei einigen Proben wurden die Aschenbestandtheile quantitativ ermittelt. Bezüglich der Dextrine wurde stets das Verhalten gegen Alkohol näher geprüft und wurden Gährungsversuche angestellt, um die Frage zu entscheiden, ob nicht die Dextrine bei Einwirkung von Hefe theilweise oder vollkommen mit dem Zucker vergähren.

Wenn ich auch heute auf eine eingehende Besprechung der erhaltenen Ergebnisse verzichten muss, so mögen doch einige Thatsachen zunächst erwähnt werden, welche der Beachtung werth erscheinen. Die Prüfung auf das Vorhandensein von grösseren Mengen von Schwefelsäure, welche man mitunter zur Erkennung von Stärkezucker in den Vordergrund stellte, ist werthlos, ebenso die Bestimmung der Alkalität der Asche der echten Honige gegenüber den Kunsthonigen, welche keine alkalische Asche liefern sollen.

Die Waldhonige sind linksdrehend und rechtsdrehend. Die rechtsdrehenden Waldhonige enthalten $0,6-1,38 \%$ Asche, die linksdrehenden $0,3-0,4 \%$. Die Asche der Waldhonige enthält $92-96^{\circ} \%$ in Wasser lösliche Bestandtheile, darunter viel lösliche Alkaliphosphate. Der Gehalt an Nichtzucker geht bei den linksdrehenden Waldhonigen bis $9,47 \%$, bei den rechtsdrehenden bis $21,2 \%$. Das spec. Gewicht der wässerigen Lösung $1: 2$ ist bei Waldhonigen grösser als bei allen übrigen untersuchten Honigen. Die griechischen Honige zeigten sehr starke Linksdrehung $\left(-13^{0}\right.$ und $\left.-15^{0}\right)$, ein Lindenhonig aus Norwegen zeigte $-17^{\circ}$, ein Heidehonig $-16^{0}$ Drehung.

Nach den bis jetzt abgeschlossenen Versuchen scheinen die Honigdextrine, besonders die der Waldhonige mit reiner Hefenkultur zum grossen Theile zu vergähren. - Der Wassergehalt ist grossen Schwankungen unterworfen, von 14-29 \% bei vollkommen reiner Waare; die Waldhonige enthalten weniger Wasser als die übrigen Honige.

Die ausführlichen Mittheilungen werden bald folgen. -

$$
\text { Diskussion. }
$$

Dr. Bömer führt aus, dass die Inversion des Zuckers zur Herstellung von Kunsthonig vielfach mit komprimirter Kohlensäure geschehe und dass daher derartige Kunsthonige keine Reaktion auf Schwefelsäure oder Oxalsäure zu zeigen brauchten. Er glaubt, dass die Bestimmung der Lecithinphosphorsäure zur Beurtheilung des Honigs von Werth sein könne und stellt die Veröffentlichung diesbezüglicher Versuche in Aussicht. Holländische Honige enthielten oft grosse Mengen zerstampfter Brut, sodass derartige Honige viel Wasser und sonstige Fremdstoffe aufwiesen. 
Bei der Bestimmung der furfurolliefernden Substanz im Pfeffer sei das Councler'sche Phloroglucin-Verfahren dem Phenylhydrazin-Verfahren nach Tollens weit vorzuziehen; letzteres sei auch von Tollens selbst schon aufgegeben.

Prof. Beckmann erklärt, dass sich die rom Vortragenden erwähnten Eigenschaften des Honigdextrins sehr wohl mit seiner Auffassung als Biose in Einklang bringen liessen. Auch die durch das Diffusionsverfahren ron $\mathrm{H}$ aenle erhaltenen Befunde liessen das Honigdextrin als Biose, also als ein Dextrin ron niedrigem Molekulargewicht erscheinen.

\title{
Zur richtigen Auslegung des Artikels „Wein“ im deutschen Arzmeibuch.
}

\author{
Von
}

W. Fresenius in Wiesbaden.

M. H.! Ich habe nur noch zu einer kurzen Mittheilung das Wort erbeten, weil mir daran liegt, einem von manchen Seiten herrortretenden Missverständnisse in Bezug auf die Auslegung des Artikels "W e in " im deutschen Arzneibuch entgegenzutreten und dadurch, dass Sie mit der von mir zu gebenden Auslegung übereinstimmen, die Richtigkeit derselben gewissermassen officiell zu bestätigen.

Wenn es in dem deutschen Arzneibuch (IV. Ausgabe) in dem genannten Artikel heisst:

„Xeres und andere Südweine, z. B. Madeira, Marsala, Gold-Malaga, Gelber Portwein, Trockenweine Ungarns, Syriens, Griechenlands, des Kaplandes und anderer Weinbaugebiete sollen in $100 \mathrm{ccm}$ nicht weniger als $11 \mathrm{~g}$ und nicht mehr als $16 \mathrm{~g}$ Alkohol, sowie nicht mehr als $8 \mathrm{~g}$ Extrakt einschliesslich des Zuckers enthalten.

An Stelle von Xeres darf zur Herstellung pharmaceutischer Zubereitungen einer der oben genannten Weine verwendet werden, wenn er auch in Farbe und Geschmack dem Xeres ähnlich ist."

so ist damit doch offenbar gar nichts anderes gemeint, als was wir in dem letzten Satze der Landshuter Beschlüsse betreffend Süssweine haben sagen wollen, wo es heisst:

Die bevorzugte Stellung der Xeres-(Sherry-)Weine im deutschen Arzneibuche ist nicht gerechtfertigt; Marsala, Port, Madeira, Goldmalaga und Capweine (als Trockenweine) können dic Xeres-Weine vollkommen ersetzen.

Es ist mit der obigen Bestimmung des Arzneibuches offenbar der damals ausgesprochene Wunsch erfüllt, es möchte festgestellt werden, welche Weine als Ersatz für Xeres zur Bereitung der "Vina composita“" verwendet werden könnten.

Es sollen dagegen zweifellos nicht die an der genannten Stelle aufgeführten Grenzen für Alkohol und Zucker für alle Süssweine gelten, welche in Apotheken verkauft werden dürfen. Es ist somit die in dem "Kommentar zum Arzneibuch für das deutsche Reich", IV. Ausgabe von B. Fischer und C. Hartwich auf S. 306 sich findende Angabe: 